\title{
Long Peripheral Catheters: A Retrospective Review of Major Complications
}

\author{
Sanjay A Patel, MD1*, Tiago Araujo, MD², Luis Parra Rodriguez, MD², \\ Claudia Ramirez Sanchez ${ }^{2,3}$, Ashley Snyder, MPH ${ }^{4}$, Vineet Chopra, MD, MSc ${ }^{4,5}$
}

\begin{abstract}
'Division of Hospital Medicine, Department of Medicine, John H. Stroger, Jr. Hospital of Cook County, Chicago, Illinois; ${ }^{2}$ Division of Post-Graduate Education, Department of Medicine, John H. Stroger, Jr. Hospital of Cook County, Chicago, Illinois; ${ }^{3}$ Department of Medicine, Rush University Medical Center, Chicago, Illinois; ${ }^{4}$ Division of Hospital Medicine, Department of Internal Medicine, Michigan Medicine, Ann Arbor, Michigan; ${ }^{5}$ A Ann Arbor Health System, Ann Arbor, Michigan.
\end{abstract}

The risk of infectious and noninfectious complications associated with long peripheral catheters (LPCs) is unknown. In this retrospective study of 539 catheters, we found LPCs were often placed for the indications of difficult access and long-term antibiotics. Rates of deep vein thrombosis $(1.7 \%)$ and catheter-related infection $(0.6 \%)$ were low. LPCs may represent a novel and safe option for short-term venous access. Journal of Hospital Medicine 2019;14:758-760. (C) 2019 Society of Hospital Medicine ntroduced in the 1950s, midline catheters have become a popular option for intravenous (IV) access. ${ }^{1,2}$ Ranging from 8 to $25 \mathrm{~cm}$ in length, they are inserted in the veins of the upper arm. Unlike peripherally inserted central catheters (PICCs), the tip of midline catheters terminates proximal to the axillary vein; thus, midlines are peripheral, not central venous access devices. ${ }^{1-3}$ One popular variation of a midline catheter, though nebulously defined, is the long peripheral catheter (LPC), a device ranging from 6 to $15 \mathrm{~cm}$ in length. ${ }^{4,5}$

Concerns regarding inappropriate use and complications such as thrombosis and central line-associated bloodstream infection (CLABSI) have spurred growth in the use of LPCs. ${ }^{6}$ However, data regarding complication rates with these devices are limited. Whether LPCs are a safe and viable option for IV access is unclear. We conducted a retrospective study to examine indications, patterns of use, and complications following LPC insertion in hospitalized patients.

\section{METHODS}

\section{Device Selection}

Our institution is a 470-bed tertiary care, safety-net hospital in Chicago, Illinois. Our vascular access team (VAT) performs a patient assessment and selects IV devices based upon published standards for device appropriateness. ${ }^{7}$ We retrospectively collated electronic requests for LPC insertion on adult inpatients between October 2015 and June 2017. Cases where (1) duplicate orders, (2) patient refusal, (3) peripheral intravenous catheter of any length, or (4) PICCs were placed were excluded from this analysis.

*Corresponding Author: Sanjay A. Patel, MD; E-mail: sanjay.a.patel@ gmail.com; Telephone: 312-864-4522.

Published online first October 23, 2019.

Received: July 2, 2019; Revised: August 19, 2019;

Accepted: August 20, 2019

๑ 2019 Society of Hospital Medicine DOI 10.12788/jhm.3313

\section{VAT and Device Characteristics}

We used Bard PowerGlide ${ }^{\circledR}$ (Bard Access Systems, Inc., Salt Lake City, Utah), an 18-gauge, 8-10 cm long, power-injectable, polyurethane LPC. Bundled kits (ie, device, gown, dressing, etc.) were utilized, and VAT providers underwent two weeks of training prior to the study period. All LPCs were inserted in the upper extremities under sterile technique using ultrasound guidance (accelerated Seldinger technique). Placement confirmation was verified by aspiration, flush, and ultrasound visualization of the catheter tip within the vein. An antimicrobial dressing was applied to the catheter insertion site, and daily saline flushes and weekly dressing changes by bedside nurses were used for device maintenance. LPC placement was available on all nonholiday weekdays from 8 AM to 5 PM.

\section{Data Selection}

For each LPC recipient, demographic and comorbidity data were collected to calculate the Charlson Comorbidity Index (Table 1). Every LPC recipient's history of deep vein thrombosis (DVT) and catheter-related infection (CRI) was recorded. Procedural information (eg, inserter, vein, and number of attempts) was obtained from insertion notes. All data were extracted from the electronic medical record via chart review. Two reviewers verified outcomes to ensure concordance with stated definitions (ie, DVT, CRI). Device parameters, including dwell time, indication, and time to complication(s) were also collected.

\section{Primary Outcomes}

The primary outcome was the incidence of DVT and CRI (Table 2). DVT was defined as radiographically confirmed (eg, ultrasound, computed tomography) thrombosis in the presence of patient signs or symptoms. CRI was defined in accordance with Timsit et al. ${ }^{8}$ as follows: catheter-related clinical sepsis without bloodstream infection defined as (1) combination of fever (body temperature $>38.5^{\circ} \mathrm{C}$ ) or hypothermia (body temperature $<36.5^{\circ} \mathrm{C}$ ), (2) catheter-tip culture yielding $\geq 103 \mathrm{CFUs} / \mathrm{mL}$, (3) pus at the in- 
sertion site or resolution of clinical sepsis after catheter removal, and (4) absence of any other infectious focus or catheter-related bloodstream infection (CRBSI). CRBSI was defined as a combination of (1) one or more positive peripheral blood cultures sampled immediately before or within 48 hours after catheter removal, (2) a quantitative catheter-tip culture testing positive for the same microorganisms (same species and susceptibility pattern) or a differential time to positivity of blood cultures $\geq 2$ hours, and (3) no other infectious focus explaining the positive blood culture result.

\section{Secondary Outcomes}

Secondary outcomes, defined as minor complications, included infiltration, thrombophlebitis, and catheter occlusion. Infiltration was defined as localized swelling due to infusate or site leakage. Thrombophlebitis was defined as one or more of the following: localized erythema, palpable cord, tenderness, or streaking. Occlusion was defined as nonpatency of the catheter due to the inability to flush or aspirate. Definitions for secondary outcomes are consistent with those used in prior studies. ${ }^{9}$

\section{Statistical Analysis}

Patient and LPC characteristics were analyzed using descriptive statistics. Results were reported as percentages, means, medians (interquartile range [IQR]), and rates per 1,000 catheter days. All analyses were conducted in Stata v.15 (StataCorp, College Station, Texas).

\section{RESULTS}

Within the 20-month study period, a total of 539 LPCs representing 5,543 catheter days were available for analysis. The mean patient age was 53 years. A total of 90 patients (16.7\%) had a history of DVT, while 6 (1.1\%) had a history of CRI. We calculated a median Charlson index of 4 (interquartile range $[I Q R], 2-7)$, suggesting an estimated one-year postdischarge survival of $53 \%$ (Table 1).

The majority of LPCs (99.6\% [537/539]) were single lumen catheters. No patient had more than one concurrent LPC. The cannulation success rate on the first attempt was $93.9 \%$ (507/539). The brachial or basilic veins were primarily targeted $(98.7 \%$, [532/539]). Difficult intravenous access represented $48.8 \%(263 / 539)$ of indications, and postdischarge parenteral antibiotics constituted $47.9 \%$ (258/539). The median catheter dwell time was eight days (IQR, 4-14 days).

Nine DVTs (1.7\% [9/539]) occurred in patients with LPCs. The incidence of DVT was higher in patients with a history of DVT $(5.7 \%, 5 / 90)$. The median time from insertion to DVT was 11 (IQR, 5-14) days. DVTs were managed with LPC removal and systemic anticoagulation in accordance with catheter-related DVT guidelines. The rate of CRI was $0.6 \%$ (3/539), or 0.54 per 1,000 catheter days. Two CRIs had positive blood cultures, while one had negative cultures. Infections occurred after a median of 12 (IQR, 8-15) days of catheter dwell. Each was treated with LPC removal and IV antibiotics, with two patients receiving two weeks and one receiving six weeks of antibiotic therapy (Table 2).

With respect to secondary outcomes, the incidence of infiltration was $0.4 \%(2 / 539)$, thrombophlebitis $0.7 \%(4 / 539)$, and

\section{TABLE 1. Patient and Device Characteristics}

\begin{tabular}{|c|c|}
\hline Characteristic & $N=539(\%)$ \\
\hline \multicolumn{2}{|l|}{ Patient } \\
\hline Age (in years), mean (SD) & $53.0(15.4)$ \\
\hline Male & $289(53.6)$ \\
\hline Body mass index, mean (SD) & $27.7(8.8)$ \\
\hline Charlson Comorbidity Index, median (IQR) & $4(2-7)$ \\
\hline History of DVT & $90(16.7)$ \\
\hline History of line infection & $6(1.1)$ \\
\hline \multicolumn{2}{|l|}{ Lab values, mean (SD) } \\
\hline INR & $1.3(0.4)$ \\
\hline PT (seconds) & $16.8(6.1)$ \\
\hline Platelets (k/uL) & $263.2(145.5)$ \\
\hline Hemoglobin (g/dL) & $12.1(31.7)$ \\
\hline PTT (seconds) & $36.8(15.0)$ \\
\hline Anticoagulant/antiplatelet administration at baseline & $147(27.3)$ \\
\hline \multicolumn{2}{|l|}{ Device } \\
\hline Dwell days, median (IQR) & $8(4-14)$ \\
\hline Single lumen & $538(99.6)$ \\
\hline 18-Gauge & $534(99.1)$ \\
\hline \multicolumn{2}{|l|}{ Inserting provider } \\
\hline Attending Faculty & $33(6.1)$ \\
\hline Vascular Access Nurse & $502(93.1)$ \\
\hline Trainee/Resident & $4(0.7)$ \\
\hline \multicolumn{2}{|l|}{ Placement attempts } \\
\hline 1 & $507(93.9)$ \\
\hline $2+$ & $32(6.1)$ \\
\hline \multicolumn{2}{|l|}{ Indication for LPC Insertion } \\
\hline IV Access & $263(48.8)$ \\
\hline Postdischarge parenteral antibiotics & $258(47.9)$ \\
\hline Chemotherapy & $17(3.2)$ \\
\hline Parenteral nutrition & $1(0.2$ \\
\hline
\end{tabular}

Abbreviations: CRI, catheter-related infection; DVT, deep vein thrombosis; INR, international normalized ratio; IV, intravenous; IQR, interquartile range; LPC, long peripheral catheter; PT, prothrombin time; PTT, partial thromboplastin time; SD, standard deviation.

catheter occlusion $0.9 \%$ (5/539). The time to event was 8.5 , 3.75 , and 5.4 days, respectively. Collectively, $2.0 \%$ of devices experienced a minor complication.

\section{DISCUSSION}

In our single-center study, LPCs were primarily inserted for difficult venous access or parenteral antibiotics. Despite a clinically complex population with a high number of comorbidities, rates of major and minor complications associated with LPCs were low. These data suggest that LPCs are a safe alternative to PICCs and other central access devices for short-term use.

Our incidence of CRI of $0.6 \%$ ( 0.54 per 1,000 catheter days) is similar to or lower than other studies. ${ }^{2,10,11}$ An incidence of $0 \%-1.5 \%$ was observed in two recent publications about midline catheters, with rates across individual studies and hospital sites varying widely. ${ }^{12,13} \mathrm{~A}$ systematic review of intravascular devices reported CRI rates of $0.4 \%$ ( 0.2 per 1,000 catheter days) for midlines and $0.1 \%$ ( 0.5 per 1,000 catheter days for peripheral IVs), in contrast to PICCs at 3.1\% (1.1 per 1,000 catheter 


\section{TABLE 2. Device-Related Outcomes}

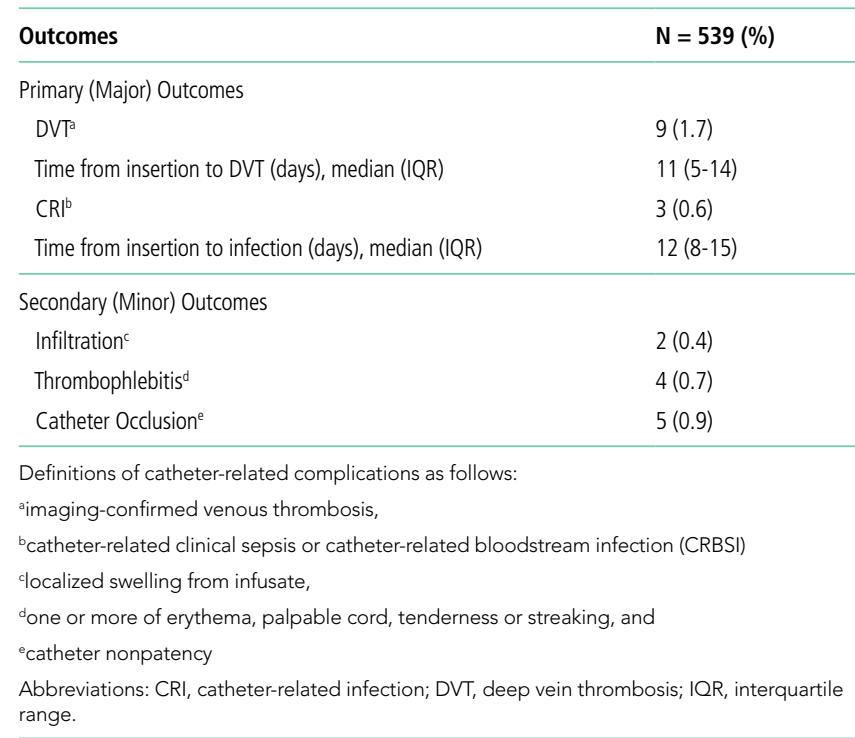

days)..$^{14}$ However, catheters of varying lengths and diameters were used in studies within the review, potentially leading to heterogeneous outcomes. In accordance with existing data, CRI incidence in our study increased with catheter dwell time. ${ }^{10}$

The $1.7 \%$ rate of DVT observed in our study is on the lower end of existing data (1.4\%-5.9\%). ${ }^{12-15}$ Compared with PICCs (2\%-15\%), the incidence of venous thrombosis appears to be lower with midlines/LPCs-justifying their use as an alternative device for IV access. ${ }^{79,12,14}$ There was an overall low rate of minor complications, similar to recently published results. ${ }^{10} \mathrm{As}$ rates were greater in patients with a history of DVT (5.7\%), caution is warranted when using these devices in this population.

Our experience with LPCs suggests financial and patient benefits. The cost of LPCs is lower than central access devices. ${ }^{4}$ As rates of CRI were low, costs related to CLABSIs from PICC use may be reduced by appropriate LPC use. LPCs may allow the ability to draw blood routinely, which could improve the patient experience-albeit with its own risks. Current recommendations support the use of PICCs or LPCs, somewhat interchangeably, for patients with appropriate indications needing IV therapy for more than five to six days. ${ }^{2.7}$ However, LPCs now account for $57 \%$ of vascular access procedures in our center and have led to a decrease in reliance on PICCs and attendant complications.

Our study has several limitations. First, LPCs and midlines are often used interchangeably in the literature. ${ }^{4,5}$ Therefore, reported complication rates may not reflect those of LPCs alone and may limit comparisons. Second, ours was a single-center study with experts assessing device appropriateness and performing ultrasound-guided insertions; our findings may not be generalizable to dissimilar settings. Third, we did not track LPC complications such as nonpatency and leakage. As prior studies reported high rates of complications such as these events, caution is advised when interpreting our findings. ${ }^{15}$ Finally, we retrospectively extracted data from our medical records; limitations in documentation may influence our findings.

\section{CONCLUSION}

In patients requiring short-term IV therapy, these data suggest LPCs have low complication rates and may be safely used as an alternative option for venous access.

\section{Acknowledgments}

The authors thank Drs. Laura Hernandez, Andres Mendez Hernandez, and Victor Prado for their assistance in data collection. The authors also thank Mr Onofre Donceras and Dr. Sharon Welbel from the John H. Stroger, Jr. Hospital of Cook County Department of Infection Control \& Epidemiology for their assistance in reviewing local line infection data.

Drs. Patel and Chopra developed the study design. Drs. Patel, Araujo, Parra Rodriguez, Ramirez Sanchez, and Chopra contributed to manuscript writing. Ms. Snyder provided statistical analysis. All authors have seen and approved the final manuscript for submission.

Disclosures: The authors have nothing to disclose.

\section{References}

1. Anderson NR. Midline catheters: the middle ground of intravenous therapy administration. J Infus Nurs. 2004;27(5):313-321.

2. Adams DZ, Little $A$, Vinsant $C$, et al. The midline catheter: a clinical review. J Emerg Med. 2016;51(3):252-258. https://doi.org/10.1016/j. jemermed.2016.05.029

3. Scoppettuolo G, Pittiruti M, Pitoni S, et al. Ultrasound-guided "short" midline catheters for difficult venous access in the emergency department: a retrospective analysis. Int J Emerg Med. 2016;9(1):3. https://doi.org/10.1186/ s12245-016-0100-0.

4. Qin KR, Nataraja RM, Pacilli M. Long peripheral catheters: is it time to address the confusion? J Vasc Access. 2018;20(5). https://doi. org/10.1177/1129729818819730.

5. Pittiruti M, Scoppettuolo G. The GAVeCeLT Manual of PICC and Midlines. Milano: EDRA; 2016.

6. Dawson RB, Moureau NL. Midline catheters: an essential tool in CLABSI reduction. Infection Control Today. https://www.infectioncontroltoday.com/ clabsi/midline-catheters-essential-tool-clabsi-reduction. Accessed February 19, 2018

7. Chopra V, Flanders SA, Saint S, et al. The Michigan Appropriateness Guide for Intravenous Catheters (MAGIC): results from a multispecialty panel using the RAND/UCLA appropriateness method. Ann Intern Med. 2015;163(6):S1S40. https://doi.org/10.7326/M15-0744.

8. Timsit JF, Schwebel C, Bouadma L, et al. Chlorhexidine-impregnated sponges and less frequent dressing changes for prevention of catheter-related infections in critically ill adults: a randomized controlled trial. JAMA. 2009;301(12):1231-1241. https://doi.org/10.1001/jama.2009.376.

9. Bahl A, Karabon P, Chu D. Comparison of venous thrombosis complications in midlines versus peripherally inserted central catheters: are midlines the safer option? Clin Appl Thromb Hemost. 2019;25. https://doi. org/10.1177/1076029619839150.

10. Goetz AM, Miller J, Wagener MM, et al. Complications related to intravenous midline catheter usage. A 2-year study. J Intraven Nurs. 1998;21(2):76-80.

11. Xu T, Kingsley L, DiNucci $S$, et al. Safety and utilization of peripherally inserted central catheters versus midline catheters at a large academic medical center. Am J Infect Control. 2016;44(12):1458-1461. https://doi.org/10.1016/j. ajic.2016.09.010

12. Chopra V, Kaatz S, Swaminathan L, et al. Variation in use and outcomes related to midline catheters: results from a multicentre pilot study. BMJ Qual Saf. 2019;28(9):714-720. https://doi.org/10.1136/bmjqs-2018-008554.

13. Badger J. Long peripheral catheters for deep arm vein venous access: A systematic review of complications. Heart Lung. 2019;48(3):222-225. https:// doi.org/10.1016/j.hrtlng.2019.01.002.

14. Maki DG, Kluger DM, Crnich CJ. The risk of bloodstream infection in adults with different intravascular devices: a systematic review of 200 published prospective studies. Mayo Clin Proc. 2006;81(9):1159-1171. https://doi. org/10.4065/81.9.1159

15. Zerla PA, Caravella G, De Luca G, et al. Open- vs closed-tip valved peripherally inserted central catheters and midlines: Findings from a vascular access database. J Assoc Vasc Access. 2015;20(3):169-176. https://doi.org/10.1016/j. java.2015.06.001. 